Sánchez Palomino, A. \& Rodríguez González, A.M. (2016). Respuesta Educativa a la Diversidad del Alumnado en Educación Secundaria Obligatoria: Análisis de los Resultados Obtenidos por las Comunidades Autónomas del Estado Español. Revista Electrónica Interuniversitaria de Formación del Profesorado, 19(3), 231-242.

\title{
Respuesta educativa a la diversidad del alumnado en Educación Secundaria Obligatoria: análisis de los resultados obtenidos por las Comunidades Autónomas del estado español
}

Antonio Sánchez Palomino, Antonio Miguel Rodríguez González Universidad de Almería

\section{Resumen}

En la presente investigación se ha realizado un estudio longitudinal sobre los resultados obtenidos por las medidas de atención a la diversidad del alumnado de Educación Secundaria Obligatoria puestas en práctica por las diferentes comunidades autónomas del Estado español. Para ello, se han enunciado una serie de hipótesis, referentes a los resultados que serían esperables obtener con la aplicación de medidas de atención a la diversidad eficientes, en aquellos colectivos concretos que son más comúnmente objeto de las mismas: alumnado con necesidades educativas especiales graves y permanentes, con discapacidad intelectual leve, con desconocimiento del idioma vehicular y con altas capacidades intelectuales. Para su corroboración, se han realizado una serie de análisis estadísticos. Los resultados obtenidos proporcionan una clasificación de comunidades autónomas según la eficiencia de sus medidas de atención a la diversidad en esta etapa educativa.

\section{Palabras clave}

Diversidad; adaptación; secundaria; eficiencia; resultados. 


\title{
Educational response to student diversity in Secondary Education: analysis of the results obtained by the Autonomous Communities of the spanish state
}

\begin{abstract}
In the present research work has been carried out a longitudinal study of the results obtained by the measures of attention to the diversity of students in Compulsory Secondary Education implemented by the different autonomous communities of the Spanish state. For this, we have set out a series of hypotheses, concerning the results that would be expected from implementation of efficient measures of attention to the diversity in the specific groups that are most commonly targets of this measures: students with severe special educational needs and permanent, with mild intellectual disabilities, with ignorance to the vehicular language and the highly gifted. For corroborate this, were performed a series of statistical analyzes. The results provide a ranking of autonomous communities according to efficiency of its measures for attention to the diversity in this educative stage.
\end{abstract}

\section{Keywords}

Diversity; adaptation; secondary; efficiency; results.

\section{Introducción}

Desde que comenzasen en España los movimientos reformistas de la Educación Especial que culminarían con la promulgación de la Ley Orgánica 1/1990, de 3 de octubre de Ordenación General del Sistema Educativo (LOGSE), hasta llegar a la actual Ley Orgánica 2/2006, de 3 de mayo, de Educación (LOE), reformada recientemente por la Ley Orgánica 8/2013, de 9 de diciembre, para la Mejora de la calidad Educativa (LOMCE); la Educación Especial en España ha experimentado un profundo cambio de concepción y modelo.

Tal y como ya exponía Marchesi (1990) en la década de los 90 del pasado siglo XX, se ha pasado de un Modelo de Educación Especial de corte Clínico, recogido en las disposiciones educativas anteriores a la LOGSE, que consideraban al alumnado con discapacidad como un sujeto con una deficiencia que necesitaba ser sometido a rehabilitación en un centro especializado y, por ende, separado del sistema educativo ordinario a, tras la aprobación de la LOGSE, un Modelo de corte Pedagógico, integrador e inclusivo, iniciado en nuestro país por autores como Toledo (1984), Fierro (1984), Marchesi y Martín (1990) y Bautista (1993), y continuado en nuestros días por, entre otros, Arnáiz (2003) o Sánchez Palomino (2001, 2011) en el cual la Educación Especial ha pasado a ser parte integrante del propio sistema educativo, dejando de estar destinada al alumnado con una discapacidad y considerándose un recurso a disposición de todo el alumnado, con el fin de dar respuesta a cualquier tipo de necesidad educativa, transitoria o permanente, ordinaria o especial, personal o debida a circunstancias socio-culturales, que el alumnado pueda presentar a lo largo de su escolarización. 
Finalmente, en la actualidad, superada la integración física del alumnado con necesidades específicas de apoyo educativo de cualquier índole en el sistema educativo, el nuevo reto consiste en alcanzar la efectiva inclusión de este alumnado.

Las distintas leyes educativas que han ido surgiendo en España hasta nuestros días, han intentado dar respuesta a una sociedad cada vez más diversa y cambiante, estableciendo una serie de medidas de flexibilización del sistema ante estas nuevas realidades: las aulas específicas, las aulas de apoyo a la integración, las adaptaciones curriculares, las aulas de adaptación lingüística, los programas de refuerzo, de diversificación curricular, de cualificación profesional inicial, etc.

Este trabajo pretende analizar los resultados obtenidos con los modelos y medidas de atención a la diversidad puestos en práctica por cada comunidad autónoma del Estado español. Para poder contrastar las diferencias existentes entre estas comunidades autónomas se formularon una serie de hipótesis iniciales, referentes a los resultados esperables si las medidas de atención a la diversidad aplicadas fuesen realmente eficaces, las cuales se han corroborado o rechazado utilizando para ello los datos aportados por las Unidades Estadísticas del Ministerio de Educación y de cada comunidad autónoma, desde el curso 2000/2001, hasta el curso 2010/11, y sometiendo dichos datos a un análisis estadístico.

En esta investigación, se ha trabajado con una premisa muy concreta, consistente en que no se han usado muestras poblacionales, sino poblaciones completas, de tal forma que las conclusiones que se alcancen sean reflejo de la realidad existente en el conjunto de España, y no circunscritas a una serie de casuísticas particulares.

\section{Método}

Para poder alcanzar los objetivos propuestos, se ha planteado una investigación cuantitativa de tipo descriptivo-evaluativa. Esta decisión se sustenta, en primer lugar, en el enorme volumen de información a manejar, ya que las fuentes informantes no serán muestras de población, sino la población completa objeto de estudio y, en segundo lugar, en que los datos a analizar en esta investigación son hechos pasados que ya han sido registrados, por lo que, como indican McMillan y Schumacher (2005), el investigador no influirá en modo alguno sobre ellos. En cambio, para poder estudiar estas variables, el investigador utiliza datos numéricos que le permitan categorizarlas cuantitativamente. Es una investigación descriptiva ya que se recopilan y analizan los resultados alcanzados por cada comunidad autónoma, con el fin de obtener una imagen lo más exacta posible de su evolución en cada variable analizada. Posteriormente, se procederá a comparar las diferencias existentes para cada hipótesis planteada entre las diferentes comunidades autónomas, buscando aquellas con un modelo de atención a la diversidad que demuestre haber obtenido, en su conjunto, mejores resultados, de ahí su naturaleza evaluativa.

\section{Población}

Las poblaciones objeto de estudio de esta investigación son las formadas por, a nivel general, el alumnado que cursó Educación Secundaria Obligatoria, desde el curso 2000/2001 hasta el curso 2010/2011 desagregado en las distintas comunidades autónomas del Estado español; y a nivel más específico, el alumnado de todo el Estado desagregado por comunidades autónomas escolarizado en Educación Secundaria Obligatoria bajo las modalidades de aula específica en centro ordinario, apoyo a la integración, el alumnado de 
compensación educativa por desconocimiento del idioma vehicular y el alumnado de altas capacidades intelectuales.

\section{Hipótesis}

Ya que esta investigación pretende describir y valorar los resultados obtenidos por las distintas comunidades autónomas durante el periodo 2000-2011 con las medidas de atención a la diversidad destinadas a los colectivos antes enumerados, el primer paso a considerar será decidir cómo se realizará dicha valoración. Para conseguirlo, se han enunciado una serie de hipótesis de partida que permitan establecer unos parámetros sobre los que poder valorar los resultados de estas medidas.

Hipótesis $n^{\circ} 1$. Con respecto al alumnado con necesidades educativas especiales graves y permanentes escolarizado en unidades específicas: Si el alumnado que asiste a estas unidades en Educación Secundaria Obligatoria alcanza un desarrollo aceptable de sus capacidades personales, debe haberse incrementado el alumnado que hayan podido acceder a un Programa de Transición a la Vida Adulta y Laboral, ya sea una F.P. de Aprendizaje de Tareas, un Programa de Garantía Social Específico o un PCPI Específico.

Hipótesis $\mathrm{n}^{\circ}$ 2. Con respecto al alumnado con discapacidad intelectual leve: Si el alumnado que asiste a las unidades de apoyo a la integración, o similares, en centros ordinarios de Educación Secundaria Obligatoria alcanza un desarrollo aceptable de sus capacidades personales, a pesar de no optar al Título de Graduado en Educación Secundaria por la vía ordinaria, podrá haber sido derivado a un Programa de Garantía Social o a un PCPI, por lo que deberá haber aumentado el alumnado de la modalidad de apoyo a la integración que accedió a estas enseñanzas.

Hipótesis $\mathrm{n}^{\circ} 3$. Con respecto al alumnado inmigrante con desconocimiento del idioma vehicular: Si el alumnado atendido en las aulas temporales de adaptación lingüística o en aulas de acogida en centros ordinarios de Educación Secundaria Obligatoria adquiere un conocimiento suficiente del idioma vehicular que garantice su incorporación exitosa a su grupo de referencia, debe haber aumentado el alumnado extranjero que obtiene el Título de Graduado en Educación Secundaria y que cursa enseñanzas postobligatorias.

Hipótesis $\mathrm{n}^{\circ} 4$. Con respecto al alumnado con altas capacidades intelectuales: Si los protocolos de detección y atención al alumnado con altas capacidades en centros ordinarios de Educación Secundaria Obligatoria son eficaces, debe haber aumentado el alumnado con altas capacidades intelectuales detectado y debe haber descendido su porcentaje de fracaso escolar, aumentando el número de matrículas en enseñanzas postobligatorias.

\section{Variables}

Para poder llevar a cabo la comprobación de estas hipótesis, se analizó la evolución diacrónica durante este periodo de las siguientes variables:

Variable $n^{\circ}$ 1: El porcentaje de alumnado de Educación Especial matriculado en Programas de Transición a la Vida Adulta y Laboral, en Programas de Garantía Social Específicos o Programas de Cualificación Profesional Específicos.

Variable $n^{\circ} 2$ : El porcentaje de alumnado escolarizado en modalidad de integración en ESO matriculado en Programas de Garantía Social y en Programas de Cualificación Profesional Inicial. 
Variable $\mathrm{n}^{\circ} 3$ : El porcentaje de alumnado inmigrante matriculado en ESO, Bachillerato y Ciclos de Formación Profesional.

Variable $\mathrm{n}^{\circ} 4$ : El porcentaje de alumnado con altas capacidades intelectuales matriculado en ESO, Bachillerato y Ciclos de Formación Profesional.

\section{Instrumentos}

Resulta necesario tener acceso a registros de matriculación tanto de las distintas enseñanzas que forman la Educación Secundaria Obligatoria (ESO, Diversificación Curricular, Programas de Garantía Social y Programas de Cualificación Profesional Inicial), como de las enseñanzas secundarias postobligatorias (Bachillerato, Ciclos Formativos de Grado Medio y de Grado Superior), referentes a los colectivos de alumnado objeto de este estudio durante el periodo 2000-2011. Para ello, se ha acudido a los datos disponibles sobre este periodo tanto en la web de la Sección de Estadística del Ministerio de Educación, Cultura y Deporte, como en las webs de las Secciones de Estadísticas de las Consejerías de Educación o los Institutos de Estadística de las diferentes comunidades autónomas, ya que, en ocasiones, los datos del Ministerio se han encontrado incompletos o no desglosados por etapas.

Especial dificultad han presentado los datos relativos al alumnado con altas capacidades intelectuales, ya que la recopilación sistemática de datos sobre este alumnado y desglosado por etapas, por parte del Ministerio de Educación, no se produjo hasta el curso 2008/2009, por lo que no se ha dispuesto del mismo número de datos para todas las comunidades autónomas, limitándose algunas al periodo 2008-2011.

\section{Procedimiento}

Una vez obtenidos los datos de población, se ha realizado el cálculo de cada una de las cuatro variables implicadas en este estudio para cada curso académico y comunidad autónoma durante el periodo 2000-2011, con el fin de poder observar su evolución y poder con ello corroborar o rechazar las hipótesis iniciales planteadas.

Para ello, aplicaremos una serie de cálculos estadísticos que permitan descartar el sesgo que podría provocar en estos resultados las simples variaciones demográficas de población escolarizada en cada etapa. Es decir, se ha pretendido descartar la posibilidad de que una determinada tendencia alcista o descendente encontrada en la evolución de alguno de los colectivos estudiados se deba simplemente al aumento o descenso de la población general que cursó dichas enseñanzas en ese mismo periodo de tiempo. Para ello, se ha calculado el coeficiente de correlación lineal, que es un estadístico que permite medir el grado de intensidad de la posible relación existente entre dos variables, que en cada caso serán la evolución de la población general que cursó determinada enseñanza y la evolución de la población del colectivo concreto que se estudia en esa hipótesis que cursó esa misma enseñanza.

En este caso se ha usado el estadístico de Coeficiente Rho de Spearman. Dicho coeficiente de correlación de Spearman, como explican McMillan y Schumacher (2005), calcula un valor de asociación de las dos variables que oscila entre -1 y +1 . Cuando su valor se acerca a +1 la relación entre ambas variables es fuerte y directa. Por tanto, cuando una variable aumente, la otra aumentará a su vez de forma similar, y viceversa. Cuando su valor se acerca a - 1 la relación entre ambas variables es fuerte e inversa. Por tanto, cuando una variable disminuya, la otra aumentará de forma similar, y viceversa. Si el coeficiente de correlación toma valores cercanos a o, la correlación entre ambas variables es débil. Por 
tanto, cuando una de ellas aumente o disminuya, la otra no variará o no lo hará en una proporción similar.

La dificultad de este estadístico no radica sólo en su cálculo, sino en determinar para cada caso a partir de qué valor se puede considerar que la Rho de Spearman calculada es o no significativa, es decir, cómo de cerca ha de estar de 1, considerado como valor absoluto, para ser significativa. Para facilitar esta labor, se ha utilizado el software estadístico S.P.S.S. (Statistical Package for the Social Sciences) de la casa IBM, el cual, además de calcular el Rho de Spearman para cada caso, indicará de forma automática si dicha correlación alcanza a ser estadísticamente significativa.

Por último, se han comparado todos los resultados obtenidos entre las distintas comunidades autónomas para la de evolución de cada variable, con el fin de localizar aquel modelo o modelos que hayan obtenido las mejores puntuaciones.

\section{Resultados}

En las siguientes Tablas se reflejan los resultados obtenidos para cada variable, desagregados por comunidad autónoma.

Tabla 1

Variaciones Porcentuales durante el periodo 2000-2011 de la Población de Unidades Específicas en centros ordinarios de Educación Secundaria, en Programas de Transición a la Vida Adulta y Laboral y en Programas de Garantía Social /Programas de Cualificación Profesional Inicial Específicos, por comunidad autónoma.

\begin{tabular}{lcccc}
\hline Comunidad & $\begin{array}{l}\text { Población de } \\
\text { Autónoma }\end{array}$ & $\begin{array}{l}\text { Población de } \\
\text { Específicas }\end{array}$ & $\begin{array}{l}\text { Población de } \\
\text { Programas } \\
\text { de TVAL }\end{array}$ & $\begin{array}{l}\text { Influencia } \\
\text { Específico }\end{array}$ \\
\hline Andalucía & +38.44 & +51.05 & -33.52 & Sí \\
Aragón & +24.76 & -25.88 & +36.57 & No \\
Asturias & +1.29 & -39.67 & -87.37 & No \\
Baleares & +23.23 & -12.82 & +12.50 & Sí \\
Canarias & +38.90 & -3.74 & -27.07 & No \\
Cantabria & +9.16 & -22.47 & -60.18 & Sí \\
C. Mancha & +27.38 & +329.57 & -19.13 & Sí \\
C. León & -6.13 & -17.87 & +7.07 & No \\
Cataluña & +8.11 & +31.17 & +111.90 & No \\
Ceuta/Melilla & +75.49 & +75 & -46.67 & Sí \\
Extremadura & +16.53 & +26.89 & +40.87 & No \\
Galicia & -18.59 & +24.50 & -38.31 & Sí \\
La Rioja & +35.59 & -13.21 & -38.10 & No \\
Madrid & +4.56 & -14.09 & +33.54 & No \\
Murcia & +50.20 & -10.09 & +54.70 & No \\
Navarra & +145.03 & +35.71 & +19.57 & Sí
\end{tabular}




\begin{tabular}{lcccc} 
País Vasco & -0.66 & -20.08 & +811.11 & Sí \\
Valencia & +7.62 & +27.32 & -38.05 & No \\
\hline
\end{tabular}

Para que se cumpliese la Hipótesis $n^{\circ}$, la población de los PTVAL y de los PGS/PCPI Específicos registrada en cada comunidad autónoma habría de haber aumentado a lo largo de la serie histórica. De haber aumentado una y descendido otra, el aumento de la primera debería como mínimo, haber compensado el descenso de la segunda. Además, dicho aumento no puede poderse explicar por un mero efecto demográfico asociado a un Rho de Spearman significativo relacionado con la población de las unidades específicas de Educación Especial en Educación Secundaria.

Pues bien, sólo en cinco comunidades autónomas se cumple dicha hipótesis, ya que aumentó el alumnado que acudió a PTVAL o PGS/PCPI Específicos sin que dicho aumento pudiese explicarse por un mero efecto demográfico. Estas comunidades autónomas, ordenadas de mayor a menor según su media aritmética entre la variación del porcentaje de población de los PTAL y de los PGS/PCPI Específicos son: Cataluña, Extremadura, Murcia, Madrid y Aragón.

Tabla 2

Variaciones Porcentuales durante el periodo 2000-2011 de la Población Escolarizada en Modalidad de Apoyo a la Integración en Educación Secundaria Obligatoria y en Programas de Garantía Social /Programas de Cualificación Profesional Inicial, por comunidad autónoma.

\begin{tabular}{lccc}
\hline $\begin{array}{l}\text { Comunidad } \\
\text { Autónoma }\end{array}$ & $\begin{array}{c}\text { Población de Apoyo a } \\
\text { la Integración en ESO }\end{array}$ & $\begin{array}{c}\text { Población de Apoyo a la } \\
\text { Integración en PGS/PCPI }\end{array}$ & $\begin{array}{l}\text { Influencia } \\
\text { demográfica (Rho) }\end{array}$ \\
\hline Andalucía & -9.97 & +57.45 & No \\
Aragón & -29.61 & +28.83 & No \\
Asturias & -12.38 & -38.27 & Sí \\
Baleares & +120.09 & +246.43 & No \\
Canarias & -64.73 & -29.58 & No \\
Cantabria & -18.03 & -34.03 & No \\
C. Mancha & -13.41 & +53 & No \\
C. León & -29.80 & -15.20 & Sí \\
Cataluña & +195.36 & +37.58 & No \\
Ceuta/Melilla & +116.67 & -35.29 & No \\
Extremadura & -47.02 & +49.15 & No \\
Galicia & +27.39 & +37.66 & No \\
La Rioja & +94.38 & +64.29 & No \\
Madrid & +37.72 & +34.85 & No \\
Murcia & +18.79 & +94.64 & No \\
Navarra & +22.75 & +47.33 & No \\
País Vasco & -22.98 & +811.11 & No \\
Valencia & -26.53 & +48.89 & No \\
\hline
\end{tabular}


Para que se cumpliese la Hipótesis $\mathrm{n}^{\circ}$ 2, la población de la modalidad de apoyo a la integración de los PGS/PCPI registrada en cada comunidad autónoma habría de haber aumentado a lo largo de la serie histórica. Además, dicho aumento no habría de poderse explicar por un mero efecto demográfico asociado a un Rho de Spearman significativo relacionado con la población escolarizada en modalidad de apoyo a la integración en ESO.

En este caso, en trece comunidades autónomas se cumple dicha hipótesis, ya que aumentó el alumnado de apoyo a la integración que acudió a PGS/PCPI sin que dicho aumento pudiese explicarse por un mero efecto demográfico. Estas comunidades autónomas, ordenadas de mayor a menor aumento de su porcentaje de población de apoyo a la integración escolarizada en PGS/PCPI son: País Vasco, Baleares, Murcia, La Rioja, Andalucía, Castilla-La Mancha, Extremadura, Valencia, Navarra, Galicia, Cataluña, Madrid y Aragón.

Tabla 3

Variaciones Porcentuales durante el periodo 2000-2011 de la Población Inmigrante en Educación Secundaria Obligatoria, Bachillerato y Formación Profesional, por comunidad autónoma.

\begin{tabular}{|c|c|c|c|c|}
\hline $\begin{array}{l}\text { Comunidad } \\
\text { Autónoma }\end{array}$ & $\begin{array}{l}\text { Población Inmigrante } \\
\text { en ESO }\end{array}$ & $\begin{array}{l}\text { Población Inmigrante } \\
\text { en Bachillerato }\end{array}$ & $\begin{array}{l}\text { Población } \\
\text { Inmigrante en } \\
\text { F.P. }\end{array}$ & $\begin{array}{l}\text { Influencia } \\
\text { demográfica } \\
\text { (Rho) }\end{array}$ \\
\hline Andalucía & +572.83 & +446.03 & +748.90 & Sí (FP) \\
\hline Aragón & +774.89 & +681.67 & +1382.29 & No \\
\hline Asturias & +736.14 & +438.36 & +851.86 & No \\
\hline Baleares & +419.46 & +551.61 & +1745.31 & Sí (FP) \\
\hline Canarias & +211.92 & +300.76 & +602.07 & No \\
\hline Cantabria & +785.20 & +631.48 & +3150 & No \\
\hline C. Mancha & +1018.60 & +1540 & +2073.44 & Sí (FP) \\
\hline C. León & +558.78 & +390.04 & +1258.73 & No \\
\hline Cataluña & +518.42 & +541.89 & +1531.32 & Sí (ESO,FP) \\
\hline Ceuta/Melilla & +273.91 & +126.19 & +3150 & Sí (ESO,FP) \\
\hline Extremadura & +326.25 & +757.14 & +653.85 & Sí (FP) \\
\hline Galicia & +421.62 & +491.95 & +1010.64 & No \\
\hline La Rioja & +1009.60 & +1090.91 & +2985.71 & No \\
\hline Madrid & +303.96 & +443.05 & +810.41 & No \\
\hline Murcia & +710.49 & +1487.93 & +1566.67 & Sí (ESO) \\
\hline Navarra & +614.03 & +537.50 & +910.87 & Sí (ESO) \\
\hline País Vasco & +610.42 & +648.26 & +1470.77 & No \\
\hline Valencia & +697.46 & +737.91 & +1424.94 & Sí (FP) \\
\hline
\end{tabular}

Para que se cumpliese la Hipótesis $n^{\circ} 3$, la población inmigrante escolarizada en estas etapas habría de haber aumentado a lo largo de la serie. Además, dicho aumento en las etapas postobligatorias no ha de poderse explicar como un efecto demográfico asociado a 
un Rho de Spearman significativo de carácter positivo entre el alumnado general de cada etapa y el alumnado inmigrante.

De esta forma, las comunidades autónomas que cumplen dicha hipótesis, ya que han experimentado aumento en su población inmigrante en ambas etapas postobligatorias sin que pueda asociarse a efecto demográfico alguno son once. Dichas comunidades autónomas, ordenadas de mayor a menor variación según la media obtenida entre la variación de población inmigrante de las etapas postobligatorias son: La Rioja, Cantabria, Murcia, País Vasco, Aragón, Castilla y León, Galicia, Navarra, Asturias, Madrid y Canarias.

Tabla 4

Variaciones Porcentuales durante el periodo 2000-2011 de la Población con Altas Capacidades Intelectuales en Educación Secundaria Obligatoria, Bachillerato y Formación Profesional, por comunidad autónoma.

\begin{tabular}{lllll}
\hline Comunidad & Población con Altas & Población con Altas & Población con & Influencia \\
Autónoma & Capacidades en ESO & Capacidades en & Altas & demográfica \\
& & Bachillerato & Capacidades en & (Rho) \\
& & & F.P. &
\end{tabular}

\begin{tabular}{lcccc}
\hline Andalucía & +461.70 & +23.09 & +1500 & Sí (FP) \\
Aragón & +6.25 & -37.50 & 0 & No \\
Asturias & +193.33 & +200 & 0 & No \\
Baleares & +53.52 & +245.45 & 0 & Sí (ESO) \\
Canarias & +40.23 & +173.33 & 0 & Sí (Bach) \\
Cantabria & +41.18 & -75 & 0 & No \\
C. Mancha & +365.38 & +400 & 0 & Sí (Bach) \\
C. León & +37.82 & -28 & 0 & No \\
Cataluña & +223.53 & +33.33 & +200 & Sí (ESO) \\
Ceuta/Melilla & +300 & 0 & 0 & No \\
Extremadura & +54.17 & +12.50 & 0 & No \\
Galicia & +63.73 & +51.85 & +100 & No \\
La Rioja & 0 & +300 & 0 & No \\
Madrid & +17.96 & +1.54 & +100 & No \\
Murcia & +3840 & +8700 & 0 & Sí (Bach) \\
Navarra & +800 & +1900 & +100 & Sí (ESO) \\
País Vasco & +32.14 & +100 & 0 & Sí (ESO) \\
Valencia & +10.53 & +90.91 & 0 & No \\
\hline
\end{tabular}

Para poder dar por cierta la Hipótesis $n^{\circ} 4$, el porcentaje de alumnado con altas capacidades intelectuales escolarizado tanto en Educación Secundaria Obligatoria como en enseñanzas postobligatorias debería haber aumentado. Como en los casos anteriores, estos aumentos de porcentaje de población con altas capacidades intelectuales no deben poderse explicar como resultado de un Rho de Spearman positivo significativo entre el 
alumnado general de cada etapa y el alumnado con Altas Capacidades Intelectuales de la misma.

Del conjunto de comunidades autónomas del Estado español, sólo en seis de ellas se cumple dicha hipótesis. Estas, ordenadas de mayor a menor variación de población con altas capacidades según la media aritmética entre las variaciones de esta población en ESO, Bachillerato y FP, son: Andalucía, Asturias, Galicia, Castilla y León, Extremadura y Madrid.

\section{Conclusiones}

Tras estas cuatro clasificaciones, derivadas de las cuatro hipótesis iniciales, se presenta el siguiente ranking de comunidades autónomas, según el grado de eficiencia demostrado por sus medidas de atención a la diversidad en Educación Secundaria Obligatoria.

Para ello, se han ordenado todas las comunidades autónomas por grupos, según el número de hipótesis que cumplen, y dentro de cada grupo, se ha colocado a cada comunidad de menor a mayor media aritmética obtenida entre los puestos que dicha comunidad alcanzó dentro de las hipótesis que cumplió, ya que resulta obvio que cuanto menor sea la media obtenida, más cerca se encontraría esa comunidad autónoma de la primera posición.

Tabla 5

Ranking de Comunidades Autónomas según su Grado de Eficiencia en la Atención a la Diversidad del Alumnado de Educación Secundaria Obligatoria.

\begin{tabular}{|c|c|c|c|c|c|c|}
\hline Orden & Comunidad Autónoma & $\mathrm{H}_{1}$ & $\mathrm{H}_{2}$ & $\mathrm{H}_{3}$ & $\mathrm{H}_{4}$ & Media \\
\hline $1^{\circ}$ & Madrid & $4^{\circ}$ & $12^{\circ}$ & $10^{\circ}$ & $6^{\circ}$ & 8 \\
\hline $2^{\circ}$ & Murcia & $3^{\circ}$ & $3^{\circ}$ & $3^{\circ}$ & - & 3 \\
\hline $3^{\circ}$ & Extremadura & $2^{\circ}$ & $7^{\circ}$ & - & $5^{\circ}$ & 4.67 \\
\hline $4^{\circ}$ & Galicia & - & $10^{\circ}$ & $7^{\circ}$ & $3^{\circ}$ & 6.67 \\
\hline $5^{\circ}$ & Aragón & $5^{\circ}$ & $13^{\circ}$ & $5^{\circ}$ & - & 7.66 \\
\hline $6^{\circ}$ & La Rioja & - & $4^{\circ}$ & $1^{\circ}$ & - & 2.50 \\
\hline $7^{\circ}$ & País Vasco & - & $1^{\circ}$ & $4^{\circ}$ & - & 2.50 \\
\hline $8^{\circ}$ & Andalucía & - & $5^{\circ}$ & - & $1^{\circ}$ & 3 \\
\hline $9^{\circ}$ & Castilla y León & - & - & $6^{\circ}$ & $4^{\circ}$ & 5 \\
\hline $10^{\circ}$ & Asturias & - & - & $9^{\circ}$ & $2^{\circ}$ & 5.50 \\
\hline $11^{\circ}$ & Cataluña & $1^{\circ}$ & $11^{\circ}$ & - & - & 6 \\
\hline $12^{\circ}$ & Navarra & - & $9^{\circ}$ & $8^{\circ}$ & - & 8.50 \\
\hline $13^{\circ}$ & Baleares & - & $2^{\circ}$ & - & - & 2 \\
\hline $14^{\circ}$ & Cantabria & - & - & $2^{\circ}$ & - & 2 \\
\hline $15^{\circ}$ & Castilla-La Mancha & - & $6^{\circ}$ & - & - & 6 \\
\hline
\end{tabular}




\begin{tabular}{llccccc}
$16^{\circ}$ & Valencia & - & $8^{\circ}$ & - & - & 8 \\
$17^{\circ}$ & Canarias & - & - & $11^{\circ}$ & - & 11 \\
$18^{\circ}$ & Ceuta/Melilla & - & - & - & - & 0 \\
\hline
\end{tabular}

Es, por tanto, la Comunidad Autónoma de Madrid la que ha presentado un modelo más eficiente de atención a la diversidad en Educación Secundaria Obligatoria durante el periodo 2000-2011, ya que ha sido la única comunidad autónoma donde se han demostrado ciertas las cuatro hipótesis planteadas en esta investigación, a modo de criterios de calidad.

En segundo lugar, queda situada la Región de Murcia, la cual, aunque presenta puntuaciones superiores a las de la Comunidad de Madrid en las tres hipótesis que le son ciertas, e incluso su grado de detección de alumnado con altas capacidades intelectuales es mayor, sus resultados para este colectivo sí se han visto afectados en la etapa de Bachillerato por fluctuaciones demográficas, lo que invalida la cuarta hipótesis planteada.

Por el contrario, el modelo de atención a la diversidad menos eficaz ha demostrado ser el diseñado por el propio Ministerio de Educación para el territorio bajo su gestión directa, es decir, las Ciudades Autónomas de Ceuta y Melilla, donde no llega a poderse demostrar como cierta ninguna de las hipótesis planteadas.

\section{Referencias}

Arjona Calvo, Y. (2011). Atención a la diversidad en Educación Secundaria Obligatoria. Estudio y propuestas para un cambio metodológico y organizativo inclusivo. Tesis para optar al Título de Doctor, Departamento de Didáctica y Organización Escolar, Universidad de Granada, Granada, España.

Arnaiz Sánchez, P. (2002). Hacia una educación eficaz para todos: La educación inclusiva. Educar en el 2000: revista de formación del profesorado, 5, 15-19.

Arnaiz Sánchez, P. (2003). Educación inclusiva, una escuela para todos. Málaga: Aljibe.

Bautista Jiménez, R. (Comp.) (1993). Necesidades educativas especiales. Málaga: Aljibe.

Blasco Calvo, P. (2006). Estrategias psicopedagógicas para la atención a la diversidad en Educación Secundaria: casos prácticos resueltos. Valencia: NAU Llibres.

Casado Melo, A. (2006). Evolución de la Atención a la Diversidad en los Textos Legislativos entre 1850 y 2000, Tesis para optar al Título de Doctor, Departamento de Ciencias de la Educación, Universidad Pontificia de Salamanca, Salamanca, España. Inédita.

Casar Domínguez, L. S. (2007). Atención a la Diversidad. Un Estudio de su Calidad en Centros de la Provincia de A Coruña. Tesis para optar al Título de Doctor, Departamento de Filosofía y Métodos de Investigación en Educación, Universidad de A Coruña, A Coruña, España. Inédita.

Chica Jiménez, M. (2005). Tutoría y Atención a la Diversidad en Educación Secundaria Obligatoria. Estudio de Casos. Tesis para optar al Título de Doctor, Departamento de Didáctica y Organización Escolar, Universidad de Sevilla, Sevilla, España. Inédita.

Espiñeira Bellón, E. M. (2008). Evaluación de la Calidad de la Atención a la Diversidad en un Centro Educativo de Galicia. Plan de Mejoras. Tesis para optar al Título de Doctor, Departamento de Filosofía y Métodos de Investigación en Educación, Universidad de A Coruña, A Coruña, España. Inédita. 
Ferrandis Martínez, M. V. (2009). La Atención a la Diversidad en Educación Secundaria Obligatoria. Tesis para optar al Título de Doctor, Departamento de Didáctica y Organización Escolar, Universidad de Valencia, Valencia, España. Inédita.

Fierro Bardaji, A. (1984). La integración educativa de escolares diferentes. Siglo Cero, 94, 1223.

López Ocaña, A. M. y Zafra Jiménez, M. (2003). La atención a la diversidad en la Educación Secundaria Obligatoria. Barcelona: Octaedro.

Marchesi Ullastres, A. (1990). Del lenguaje de las deficiencias a las escuelas inclusivas. En Coll, C. Marchesi, A. y Palacios, J. (1990), Desarrollo Psicológico y Educación. Vol. 3 (pp. 2144). Madrid: Alianza Editorial.

Marchesi Ullastres, A. y Martín Ortega, E. (1990). Del lenguaje del trastorno a las necesidades educativas especiales. En Coll, C. Marchesi, A. y Palacios, J. (1990), Desarrollo Psicológico y Educación. Vol. 2 (pp. 15-33). Madrid: Alianza Editorial.

MCMillan, J. H. y Schumacher, S. (2005). Investigación Educativa. Madrid: Pearson Educación.

Ministerio de Educación (1990). Ley Orgánica 1/1990, de 3 de octubre, de Ordenación General del Sistema Educativo. Madrid: Autor.

Ministerio de Educación (2006). Ley Orgánica 2/2006, de 3 de mayo, de Educación. Madrid: Autor.

Ministerio de Educación (2007). Orden ECl/2220/2007, de 12 de julio, por la que se establece el currículo y se regula la ordenación de la Educación Secundaria Obligatoria. Madrid: Autor.

Ministerio de Educación (2013). Ley Orgánica 8/2013, de 9 de diciembre, para la Mejora de la Calidad Educativa. Madrid: Autor.

Miñan Espigares, A. (Coord.) (2006). Experiencias de atención a la diversidad en Educación Secundaria. I Jornadas Andaluzas sobre Experiencias de Atención a la Diversidad en Educación Secundaria. Granada. España.

Otero Fernández, M. J. (2011). Medidas de Atención a la Diversidad en la Enseñanza Secundaria Obligatoria en los centros del Sector Educativo de Santiago. Tesis para optar al Título de Doctor, Departamento de Didáctica y Organización Escolar, Universidad de Santiago de Compostela, Santiago de Compostela, España. Inédita.

Rodríguez Tejada, R. M. (2009). La Atención a la Diversidad en la ESO. Actitudes del Profesorado y Necesidades Educativas Especiales. Badajoz: Diputación de Badajoz.

Sánchez Palomino, A. (2001). De la integración a la escuela para todos. Granada: Grupo Editorial Universitario.

Sánchez Palomino, A. y Otros (2011). Educación Especial y Mundo Digital. Almería: Universidad de Almería.

Toledo González, M. (1984). La escuela ordinaria ante el niño con necesidades especiales. Madrid: Santillana. 